\title{
Motor Control for Wind Tunnel Precision Speed Regulation for the Wind Tunnel Motor at California Institute of Technology
}

\author{
BY WILLIAM A. LEWIS \\ Associate, A. I. E. E.
}

\begin{abstract}
Synopsis. - A wind tunnel for testing model airplanes and their parts requires accurate control of the air velocity. This paper describes a tunnel having electric drive for producing the air movement and explains a system of control, which allows a wide range of
\end{abstract}

speeds and holds the speed very constant at any set value. Either hand or automatic regulation may be employed. The hand control is used for fairly constant speed while the automatic control gives very close regulation.

\section{INTRODUCTION}

$\mathrm{T}$ $\mathrm{HE}$ widespread interest in aviation developed during the last few years has resulted in a large increase in the facilities both for teaching aeronautics and for carrying on further investigations in this field. Under the terms of a grant from the Daniel Guggenheim Fund for the Promotion of Aeronautics, a graduate school of aeronautics was recently established at the California Institute of Technology. One of the principal features of the laboratory, built for the purpose of carrying on the experimental work in this department, is a high-speed wind tunnel with a working section ten feet in diameter. The propeller which forces the air through the tunnel is electrically driven, and the equipment and its control present several interesting features, which will be described in this paper.

\section{WiND TUNNELS}

Before proceeding to a discussion of the drive, a general description of the tunnel and its use would be desirable. Wind tunnels are used for testing model airfoil sections and new plane designs to determine performance, in the case of planes particularly with regard to taking off and landing. The model to be tested is supported in the center of the working section, usually in an inverted position, and when a stream of air passes the model, the relative motion of air and model simulate flying conditions. The model is supported by wires attached at three points and is held in position by the wires and a series of counterweights. The supporting wires are attached to a set of balances either directly or, as in this case, through a subframe. The reactions of the model may be separated into two components, a force downstream or drag, and an upward force or lift. Since the model is inverted, the upward force with respect to the model is downward with respect to the balances. These forces are instantly felt at the balances and can be computed from the balance readings. The values of the forces together

1. Formerly Teaching Fellow, California Institute of Technology; General Engineer, Westinghouse Electric \& Mfg. Co., East Pittsburgh, Pa.

Presented at the Pacific Coast Convention of the A.I.E.E., Santa Monica, Calif., Sept. 3-6, 1929. with the temperature, pressure, and velocity of the air, are the data for determining the performance of the model.

This tunnel is of the closed-circuit type, the same air being recirculated. A longitudinal section is shown in Fig. 1. The tunnel occupies a height of nearly four floors, the over-all vertical dimension being about $45 \mathrm{ft}$. It consists of sections of circular cylinders and cones, connected end-to-end to form the closed circuit shown in the illustration. The four sections in the observation room are made of redwood staves held together by hoops of steel rod and angle iron on the outside. If desired, one or more of the sections may be removed and the tunnel operated with open throat, the circuit being closed by the observation room itself. The remainder of the tunnel is made of reinforced concrete, the interior surface of which was formed by the Gunnite spraying process. At the intersections of the vertical and horizontal sections a series of deflecting vanes changes the direction of the wind with minimum loss of energy. The completed vane installation in the lower 20 -ft. intersection is shown in Fig. 2. The vanes in the two left-hand corners are arranged so that at a future date, cooling water may be circulated through them to assist in cooling the air in the tunnel. The entire tunnel is supported on its own foundation free from the building, to minimize transmission of vibration.

\section{WIND VeLOCITY REQUIREMENTS}

For preliminary work it is desirable to control the velocity of the wind from the observation room, but for accurate testing the performance is determined entirely from the balance readings so that the balance operator must have instant and accurate control over the propeller speed. Because of the many variables involved, engineering accuracy requires that the variation in each, particularly wind speed, be kept as small as possible. The maximum allowable variation in propeller speed for satisfactory operation is \pm 0.25 per cent. At the same time, in order to make a complete series of tests it is necessary that the air speed be adjustable over a wide range.

To fulfill these requirements the equipment described below was designed and installed. With it, 
any wind velocity past the model from a slight breeze produced by a propeller speed of only about $40 \mathrm{rev}$. per min. to a cyclone of approximately $200 \mathrm{mi}$. per $\mathrm{hr}$. at a propeller speed of 850 rev. per min., can be easily obtained by operating a single control, located at any point desired. Within the range from 130 to $850 \mathrm{rev}$. per min. the speed control can be transferred to a regulator which will maintain the speed constant with a

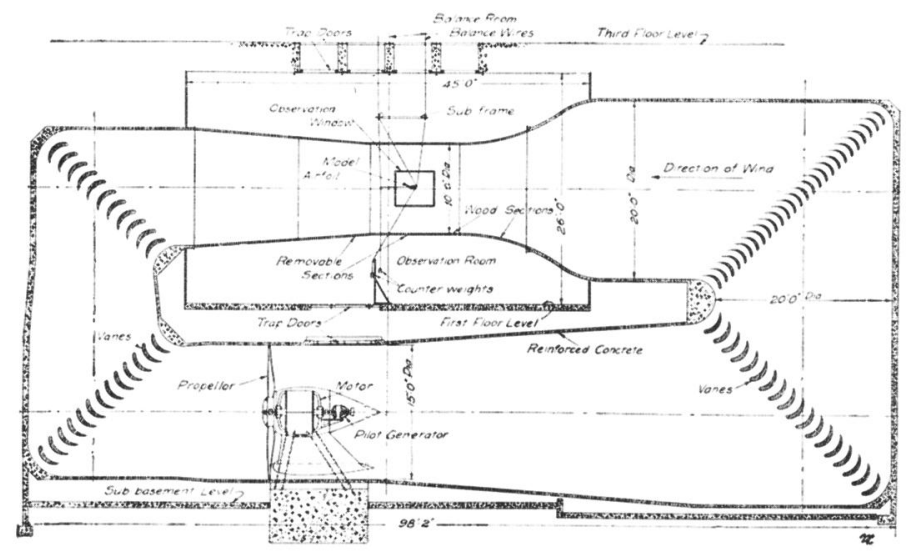

Fig. 1-Longitudinal Section of the Wind Tunnel (Vertical Plane)

very high degree of accuracy. This range corresponds to air velocities of 10 to $200 \mathrm{mi}$. per hr. By adjusting the positions of a coarse and fine rheostat, one of which is located at each station, the speed held by the regulator may be easily adjusted to any value in its range.

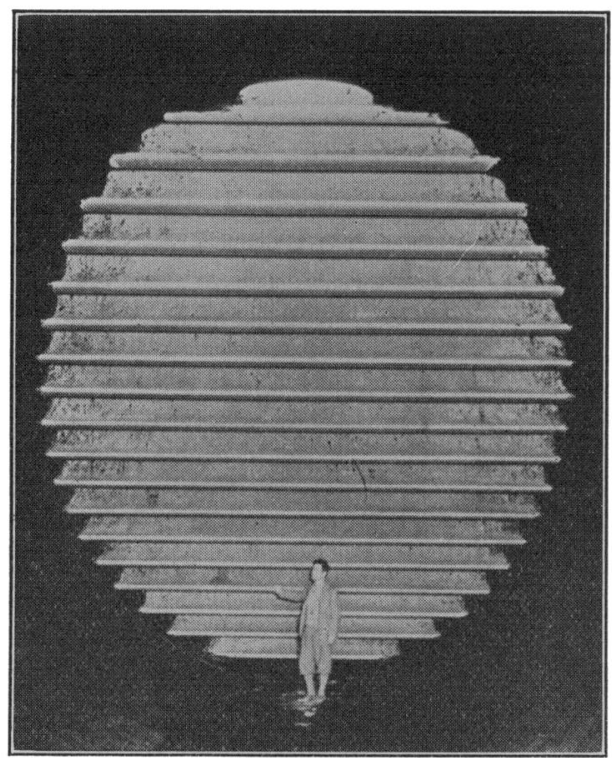

Fig. 2-One of the Lower Deflecting Vane Installations

\section{Profeller MOTOR}

The propeller is made with four detachable blades mounted in a central cast hub. The diameter of the propeller is $14 \mathrm{ft} .11 \mathrm{in}$. and of the tunnel at the section where the propeller is located approximately $15 \mathrm{ft}$., so that clearance between the propeller and the tunnel wall is very small. After consideration of all advantages and disadvantages, it was decided to place the motor inside the tunnel, supporting it on a structural steel frame extending out through the walls of the tunnel into a heavy concrete base, and to mount the propeller directly on the end of the motor shaft. The obstruction introduced is not serious if the motor and its framework are not too large, since aerodynamical considerations require that the wind stream be contracted just beyond the propeller.

To drive the propeller at the maximum speed requires an input of approximately $750 \mathrm{hp}$. However, the time for obtaining a set of readings is not great and it was estimated that a machine of $500 \mathrm{hp}$. continuous capacity with short-time overload ratings, would be satisfactory. The standard machine of this size is equipped with bed-plate and pedestal bearings, and was not well adapted for the type of mounting required. A special

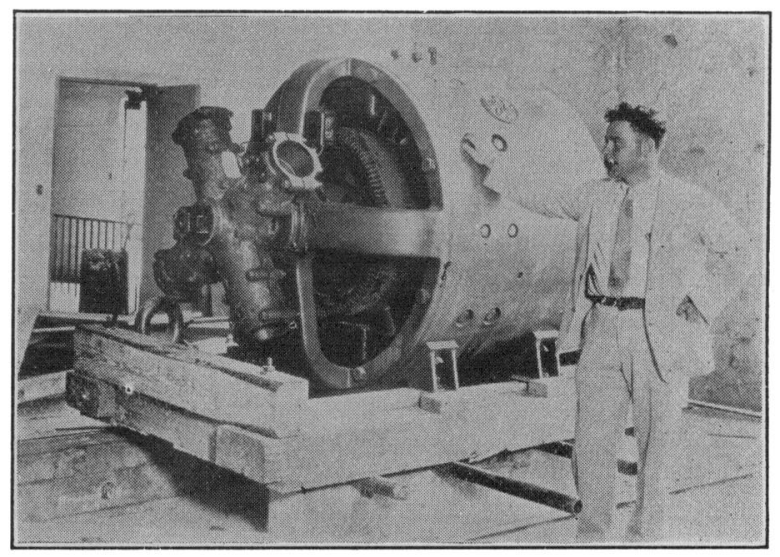

Fig. 3-Propeller Motor, 500 Hp. Continuous Rating

design, with bearings mounted in end brackets supported directly from the motor frame, developed for submarine and other transportation purposes, solved the problem. In this way a motor having a completely cylindrical frame and an over-all diameter of only $4 \mathrm{ft} .8 \mathrm{in}$. was obtained. The motor, before installation, is shown in Fig. 3. The feet shown in the figure were used for transportation only, it being found that a smaller overall diameter of motor and covering would result if the feet were eliminated, and the supporting framework made to fit the motor frame. Because of the errors which would be introduced into the speed regulation by vibration, the framework was made very heavy and rigid so that its natural frequency is far higher than any introduced by the propeller. A semi-cylindrical steel-plate cradle fits the lower half of the motor frame and is bolted directly to the motor. Four heavy $\mathrm{H}$-section columns form the supporting legs and are riveted to the cradle. In assembling, the heavily reinforced concrete base for the framework was first cast complete, with the exception of four pockets for the legs. The legs were then inserted in these pockets, and 
the cradle riveted to the legs. Next the motor was mounted, the entire structure alined as a unit, and the pockets filled with concrete to unite the framework and base into a continuous whole. The U-shaped pockets in the rear $\mathrm{H}$-section legs were covered with steel plates and used for wiring gutters, cored ducts in the concrete base forming a continuation of these gutters to accessible locations. The details of the structure can be seen in Figs. 4 and 5.

\section{COOLING THE MotoR}

One serious problem, introduced by mounting the motor inside the tunnel, is that of securing adequate

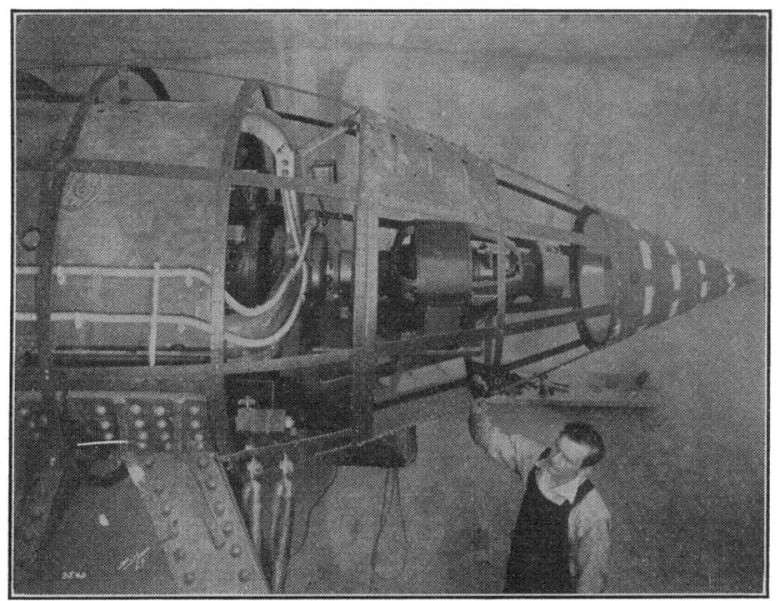

Fig. 4-Propeller Motor and Pilot Generator Mounted in the Tunnel and Partly Covered by Stream Line Fairing

ventilation and cooling. The entire output of the propeller is eventually converted into heat by friction of the air, and since the air is recirculated, this heat, together with that due to the losses in the motor, will be taken up by the air and walls of the tunnel. As data for determining the heat transfer from the air to the tunnel walls were meager and inaccurate, it was impossible to predict the temperature to which the air would rise or the time required to reach equilibrium. If the tunnel air remained within reasonable temperature limits, it could be used for cooling the motor, but an air temperature much in excess of 50 deg. cent. would make a separate cooling system necessary. Several estimates placed the average air temperature in the tunnel at 45 deg. cent. Because of the expense of external cooling and in view of the difficulty of carrying on work in the observation room when the temperature of the tunnel is excessive, it was felt that use of the tunnel air for motor cooling would be satisfactory. Part of the air from the propeller is deflected through the motor air passages.

In order to keep the friction loss caused by the air passing outside the motor at a minimum, it was necessary to enclose the motor and its support in a stream line fairing, broad nosed at the propeller end and tapering off to a point at the tail. An opening in the nose and louvres in the sides allow the ventilating air to pass through the motor. In order to keep the pilot generator, (a small generator connected to the main shaft and used with the speed regulator) at as constant a temperature as possible, it is ventilated separately. A baffle inserted between the two machines produces the desired result. A second set of louvres behind the baffle and a third set in the tail provide the necessary cooling air circulation. The fairing is composed of a skeleton framework, Fig. 4, covered with steel plates screwed in place. The section covering the propeller hub revolves with the propeller but the remainder is stationary. To obtain access to any part of the motor it is necessary merely to remove the adjacent plates. An ingenious assembly of the skeleton frame allows a large section of the fairing to be removed as a unit in case of major repairs. Views of the fairing framework with several of the plates mounted are shown in Figs. 4 and 5 . After the fairing of the motor itself had been completed, the legs on each side of the shaft were enclosed in additional fairings. Views of the completed installation are shown in Figs. 6 and 7.

\section{Motor-Generator Set}

The propeller motor is a d-c. commutating pole machine, since such a speed range could not be obtained with constant frequency alternating current. For furnishing the direct current and providing a simple means of speed variation, an individual synchronous

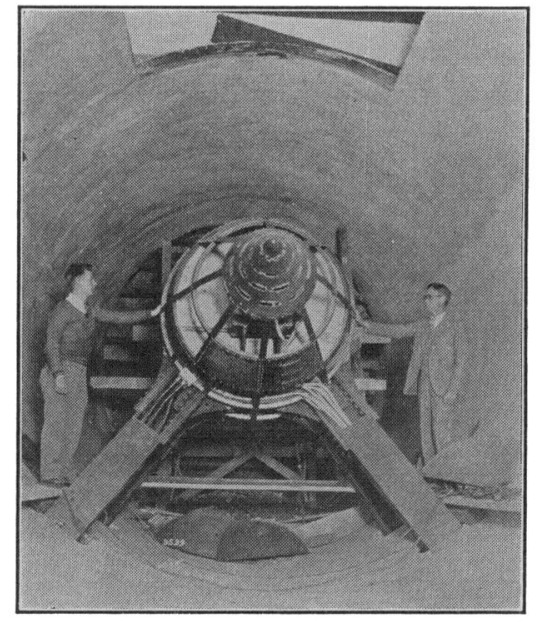

Fig. 5-End View of Motor Showing Supporting Legs Used For Wire GutTers

motor-generator set is provided. In order to make available the maximum possible amount of direct current for physical experiments, the nominal d-c. voltage was established at 230 volts, although the actual voltage varies from residual of the generator up to about 300 volts, depending upon the speed of the propeller motor. Both the synchronous motor and the d-c. generator are provided with direct-connected exciters, separate machines being required because of the speed regulator.

\section{METHOD OF CONTROL}

As the motor-generator set had to be located quite 
close to the propeller motor, because of the large currents involved, it was placed in the sub-basement of the building, just outside the tunnel. The most desirable location for the control station being near the balances in the balance room, five floors above, electrical remote control was adopted for all equipment requiring oper-

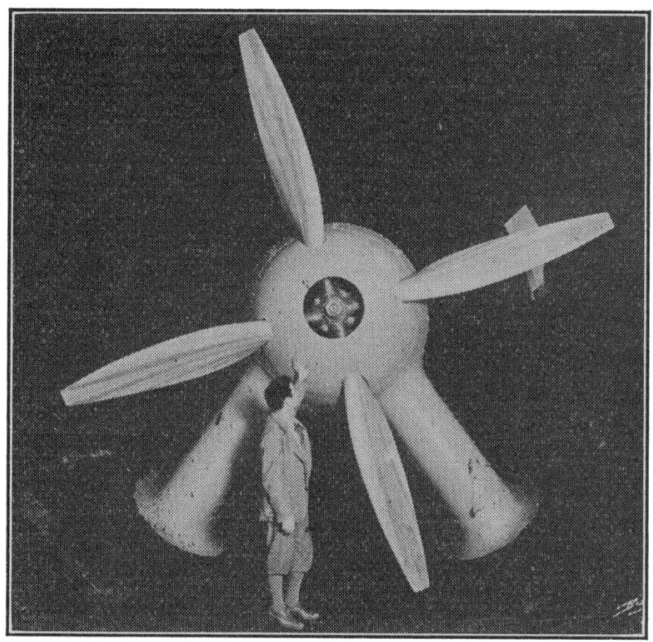

Fig. 6-Propeller End of Main Motor with Fairing Completed

ation during normal running of the tunnel. Direct current was considered the most suitable for control power, and continuity of service not being absolutely essential, a small induction motor-generator set, giving 125 volts direct current was provided instead of the more expensive storage battery. The former type of

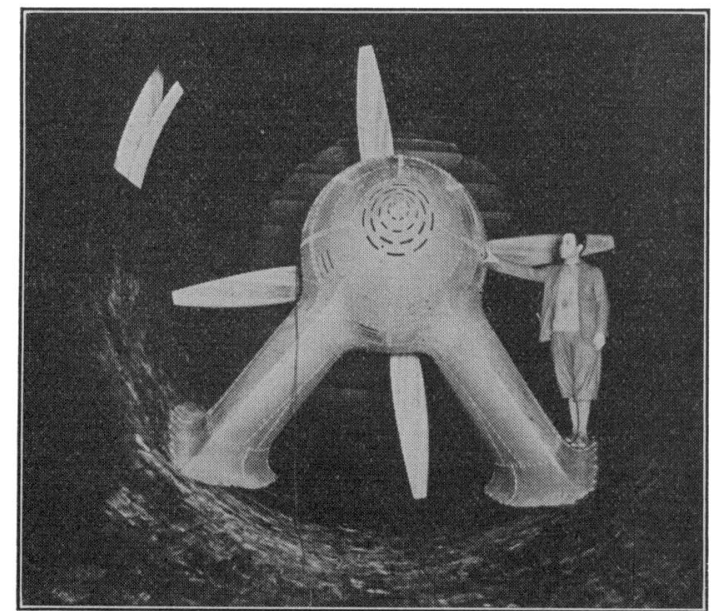

Fig. 7-Down Stream End of MaIN Motor Showing Completed Installation

control, however, is not absolutely reliable and provision had to be made for disconnecting all the main machines upon failure of control voltage, thus complicating the control circuits considerably.

The apparatus for starting and controlling the a-c. end of the motor-generator set is practically standard automatic equipment and need not be described here. However, since the propeller motor is neither visible nor audible from the point of normal operation and the operators are in general non-electrical men, it was considered worth while to design the entire installation for unattended operation and most of the features common to automatic stations, such as lock-out relaybearing temperature relays, reverse-phase voltage, current relays, etc., are provided. Also the switching operations of starting are automatically controlled so that the field is applied and the transfer to full voltage is accomplished without the intervention of the operator.

To secure the wide speed range necessary for the propeller motor, the armature voltage or Ward Leonard system of control is necessary. To obtain good efficiency and regulation with this system it is necessary to vary the voltage of the generator which supplies the motor armature. Control is exercised in this case by

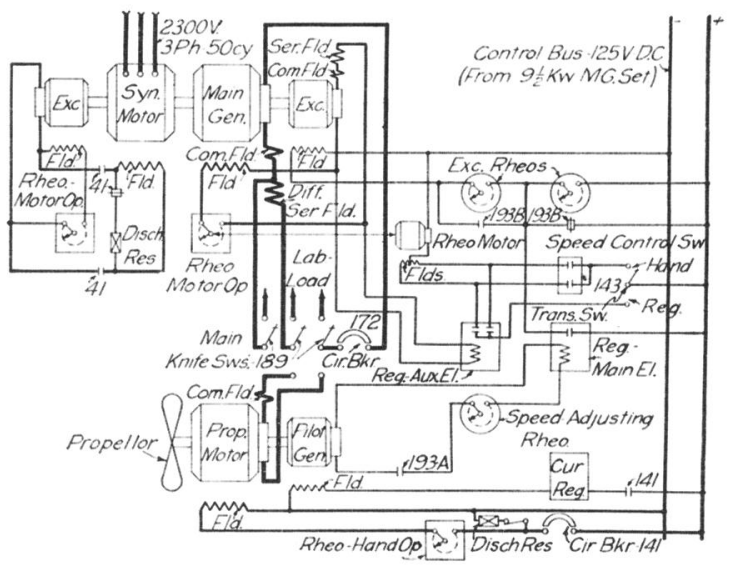

Fig. 8-Schematic D-c. Circuits for Automatic Speed Control and Power Supply

varying the excitation of the generator either by changing the position of the generator field rheostat or the voltage of the exciter or both. The field of the motor must, of course, be supplied from a separate constant voltage source and in this case was connected to the control generator because the voltages of both direct-connected exciters for the motor-generator set are subject to excessive changes. This connection also resulted in the smallest installed auxiliary capacity, since field for the propeller motor is not required until all the large oil circuit breakers have been closed and the closing solenoids deenergized, thus making possible joint use of the same control-generator capacity. The main d-c. connections are shown in Fig. 8, and the armature and field circuits may be easily traced on the diagram. It will be noticed that a series of doublethrow switches is inserted in the armature circuit of the main generator and that the two right-hand switches must be closed in the lower position in order to connect the propeller motor. Under these conditions the differential series field is in circuit, acting to stabilize the speed by opposing a change in current. When the 
generator is used for other experiments, the differential field may or may not be desired and the third switch allows a choice to be made.

It may also be noticed that no starting resistance is provided in the propeller-motor circuit. The starting current is kept within sufficiently low limits by reducing the generator voltage to its minimum value before closing circuit breaker 172 . It is also necessary, of course, that the field circuit be closed before the armature circuit is closed, and that the armature circuit open whenever the field circuit opens. These conditions are obtained by means of suitable interlocks so that circuit breaker 172 cannot be closed until the motor-operated rheostat is "all in," and as soon as the circuit breaker opens, connections not shown in the diagram run the rheostat to this position. The motor is started by operating a single control switch. If the rheostat is in the proper position, the motor starts immediately. If not, the motor will start as soon as the rheostat reaches the "all in" position. The connections are arranged so that field circuit breaker 141 closes first, followed by the closing of circuit breaker 172. When stopping, whether by hand or by one of the protective features, the breakers open in the reverse order. If the field breaker opens for any reason, the armature circuit breaker opens immediately.

After the motor has been started, two types of speed control are available. Under hand control the speed of the motor is brought to the desired value and remains nearly constant due to the inherent regulation of the system, although small variations and a gradual creep in speed, due to temperature effects, occur. However, this type of control is much simpler and is very satisfactory for rough or preliminary work. Under regulated control the speed is set at a given value corresponding to the position of the speed adjusting rheostat, consisting of a coarse and fine section, separately adjustable, and is held at that speed with a high precision by the speed regulator. Although several control stations of both types are provided, only one of each is shown in the diagram. To accommodate additional stations the number of positions of transfer switch 143 is increased, and additional speed adjusting rheostats and relays, 193, are provided for each regulated station and additional speed control switches for each hand control station. For hand control, the entire adjustment is obtained by control of the generator field rheostat, whereas the two elements of the regulator control both the voltage of the exciter and the position of the generator rheostat.

For hand control, transfer switch 143 is closed in the hand position, thus connecting the speed-control switch to a source of power, and the operation of this switch will cause the rheostat to be driven in one direction or the other, thus increasing or decreasing the generator voltage and consequently the speed of the propeller motor. It may be noticed that there are two rheostats in the exciter field circuit, so that the voltage of the ex- citer is dependent on the position of both rheostats. However, under hand control, relay $193-\mathrm{B}$ is deenergized so that its back contact is closed, short-circuiting the right-hand rheostat, and the exciter voltage is thus determined in this case entirely by the position of the left-hand rheostat. The latter is set at a position which will give normal exciter voltage and is left unchanged, so that the generator rheostat has complete control of the propeller-motor speed.

To obtain regulated control, transfer switch 143 is changed to the "Reg." position. Auxiliary circuits, not shown, immediately run the generator rheostat to the "all in" position, and energize relays 193-B and 193-A. Relay 193-A connects the pilot generator armature to the main element of the speed regulator through the speed adjusting rheostat. Relay 193-B short circuits the left-hand exciter rheostat and inserts the right-hand one in the circuit. The field of the pilot generator is energized through an auxiliary contact of the field circuit breaker 141 and the current regulator, and therefore carries a constant current whenever the propeller motor is running. Under these conditions the voltage generated by the pilot generator is directly proportional to speed. A definite fraction of this voltage, depending upon the position of the speed adjusting rheostat, is impressed upon the main element of the regulator. If this voltage is below the amount required to open the regulator contacts, the contacts close and short-circuit the exciter rheostat. This raises the exciter voltage, hence also the generator voltage, the motor speed, and the pilot generator voltage. If the increase in pilot generator voltage is sufficient, the contacts will open, the exciter voltage will fall and with it, the generator voltage, the propeller motor speed, and the pilot generator voltage, until the contacts close again. The process repeats continuously. An auxiliary coil and plunger, not shown in the diagram but connected across the exciter terminals, react on the contacts in the same manner as the d-c. coil in an a-c. generator voltage regulator, preventing hunting and keeping the oscillations in speed, produced by the above described opening and closing of contacts, within the most minute limits. Under this condition the mean speed is a function of the position of the speed adjusting rheostat, since the average current in the regulator coil must be a constant and the drop across the rheostat then depends only on its position.

If, in the first instance, the increase in pilot generator voltage was insufficient to open the contacts, the vibrating action does not occur and a speed balance is not obtained. The speed range over which a balance can be obtained with a fixed position of the generator field rheostat is very small, and to increase this range, the auxiliary element of the regulator is provided. If the main contacts remain closed, the exciter voltage will rise above a predetermined value at which one of the contacts of the auxiliary element closes, and this contact changes the position of the generator rheostat until the 
regulator contacts open, the exciter voltage falls, and the auxiliary contact opens again. Conditions are now satisfactory and the vibrating action of the contacts is immediately set up and a balance obtained. In case the resistance in the speed-adjusting rheostat is reduced, the regulator contacts immediately open, reducing the exciter voltage, the generator voltage, the motor speed, and the pilot generator voltage in turn. If the drop is sufficient, the contacts will close again, a new vibrating action will be set up and a new speed maintained. If the contacts do not close, the exciter voltage will fall so low that the other contact of the auxiliary element will close, increase the resistance in the generator field, reduce the speed of the propeller and finally the pilot generator voltage, until the contacts again close and a new vibrating point is established. Thus, the quickacting vibrating regulator maintains a precision control over a narrow range and this range is shifted to the proper part of a much broader range by means of the auxiliary element, thus providing a precise speed control over a broad range.

\section{OPERATING RESUlTS}

The installation described above is giving entire satisfaction in the operation of the wind tunnel at California Institute of Technology, both with regard to ease of control and accuracy of speed regulation. Although no precision instruments are available for measuring the actual instantaneous variations, observations of an accurate electric tachometer indicate instantaneous variations of less than 0.2 per cent plus or minus, after the regulating equipment has assumed operating temperature, requiring about one half-hour.

There are innumerable causes of instantaneous speed variation, the principal ones being resistance changes due to temperature variations in the armature and field of the propeller motor, main generator and exciters, change in load due to change in angle of attack of the model, change of supply frequency, etc. However, none of these changes can exceed the limit given above of \pm 0.2 per cent without bringing about a corrective action from the regulator, so that none of these effects produce any permanent variations or any beyond the stated limits unless they are of such extreme magnitude and so rapid that the regulator is unable to respond and correct them before the limit is reached.

The only causes of permanent variation are those which affect the accuracy of the speed regulator, and include changes in resistance of the regulator circuits and change in permeability of the pilot-generator field with temperature. Such effects cause a gradual increase in speed of approximately 0.25 per cent per hour after operating temperature has been reached, and may be easily corrected for by adjustment of the speed-adjusting rheostat.

As explained above, no permanent speed change is caused by variation in a-c. line voltage or frequency, the changes being immediately corrected for by action of the speed regulator. No data are available regarding the speed of corrective action of the regulator or the amount and rate of change necessary to prevent the regulator keeping the speed within the allowable limits. Although the assembly was developed for a special application, it is composed entirely of standard apparatus and has several features which may be adaptable to other purposes requiring a wide speed range with a high degree of accuracy in speed regulation.

\section{ACKNOWLEDGMENT}

The author wishes to acknowledge the kind cooperation of many members of the staff of the California Institute of Technology, especially Mr. L. G. Fenner, Superintendent of Wiring; also of many members of the engineering staffs of the Westinghouse Electric \& Manufacturing Company and General Electric Company.

\section{Appendix}

\section{Apparatus}

Propeller Motor: 500-hp., 230-volt, 700-rev. per min., at full load, shunt connected.

Main Generator: 430-kw., 230-volt, 1000-rev. per min., differential compound.

Main A-C. Motor: 610-hp., 2200-volt, 3-phase synchronous.

M-G Set for Control Supply: 91/2-kw., 125 volt, 1500-rev. per min. compound generator; 220-volt, 3-phase induction motor.

Pilot Generator on the Propeller Motor Shaft: Rated $1.5 \mathrm{kw}$. (but in a larger frame for negligible temperature rise) 600-volt, 700-rev. per min., separately excited.

Automatic Switchboard contains: 3000-ampere automatic circuit breaker, accelerating relays, misc. relays, field contactor, overload relays, annunciator relay.

Speed Control Switchboard contains: Phase balance relay, voltage balance relay, control switches and indicators and regulator operating from the pilot generator.

Much additional apparatus such as exciters and switchboard meters have not been listed in detail. 